\title{
Absceso del uraco y obstrucción intestinal en paciente con obesidad mórbida: reporte de caso
}

\author{
Urachal abscess and intestinal obstruction in a morbidly obese patient: \\ A case report
}

Paulo Andrés Cabrera-Rivera ${ }^{1} \mathbb{D}$, Bayron Rafael Guerra Benedetti ${ }^{2} \mathbb{D}$, Carlos J. Pérez-Rivera ${ }^{\mathbb{D}}$, Lina Sanabria ${ }^{4} \mathbb{D}$, Sara Sofía Avendaño ${ }^{5}$ D, Paula Sanabria-Torres ${ }^{5} \mathbb{D}$

1 Médico, especialista en Cirugía general, candidato a Magister en Epidemiología. Jefe del Departamento Cirugía General, Fundación Cardionfantil - Instituto de Cardiología, Bogotá, D.C., Colombia.

2 Médico, especialista en Cirugía general, Fundación Cardionfantil - Instituto de Cardiología, Bogotá, D.C., Colombia.

3 Médico, especialista en Epidemiología, candidato a Magister en Epidemiología, Grupo de investigación Cirugía general, Fundación Cardionfantil - Instituto de Cardiología, Bogotá, D.C., Colombia.

4 Médico, residente de Cirugía general, Grupo de investigación Cirugía general, Fundación Cardionfantil - Instituto de Cardiología, Bogotá, D.C., Colombia.

5 Médico, Grupo de investigación Cirugía general, Fundación Cardionfantil - Instituto de Cardiología, Bogotá, D.C., Colombia.

\section{Resumen}

Las anomalías del uraco representan un bajo porcentaje de las patologías abdominales, no obstante, forman parte del diagnóstico diferencial del abdomen agudo por las complicaciones que puede tener. Aunque son de difícil diagnóstico debido a los síntomas inespecíficos, las imágenes diagnósticas son de gran utilidad para su identificación y caracterización. En pacientes con obesidad mórbida, la presentación del cuadro aumenta el riesgo de morbimortalidad. Por ende, en estos pacientes es necesario un control postoperatorio estricto para evaluar complicaciones secundarias a la resección del uraco. Dado los casos limitados en la literatura, se requieren estudios clínicos adicionales, para brindar un seguimiento adecuado, en aras de identificar complicaciones y el tratamiento precoz de estas.

Palabras clave: obesidad mórbida; obstrucción intestinal; quiste del uraco; uraco.

\begin{abstract}
Urachal abnormalities represent a low percentage of abdominal pathologies; however, they are part of the differential diagnosis of acute abdomen due to the complications it may have. Although they are difficult to diagnose due to nonspecific symptoms, diagnostic images are especially useful for their identification and characterization. In patients with morbid obesity, the presentation of the picture increases the risk of morbidity and mortality. Therefore, in these patients, strict postoperative control is necessary to evaluate complications secondary to urachal resection. Given the limited cases in the literature, additional clinical studies are required to provide adequate diagnosis and follow-up in order to identify complications and their early treatment.
\end{abstract}

Keywords: morbid obesity; intestinal obstruction; urachal cyst; urachus.

Fecha de recibido: 12/01/2020 - Fecha de aceptación: 27/04/2020- Fecha de publicación en línea: 05/03/2021

Correspondencia: Carlos J. Pérez-Rivera, Calle 163A \# 13B - 60, Departamento de Investigaciones, Fundación Cardioinfantil - Instituto de Cardiología, Bogotá, D.C., Colombia. Teléfono: 6672727 ext. 73203. Correo: cjperezrivera@gmail.com

Citar como: Cabrera-Rivera PA, Guerra BR, Pérez-Rivera CJ, Sanabria L, Avendaño SS, Sanabria-Torres P. Absceso del uraco y obstrucción intestinal en paciente con obesidad mórbida: reporte de caso. Rev Colomb Cir. 2021;36:352-7. https://doi.org/10.30944/20117582.511 Este es un artículo de acceso abierto bajo una Licencia Creative Commons - BY-NC-ND https://creativecommons.org/licenses/by-ncnd/4.0/deed.es 


\section{Introducción}

El uraco es un remanente embriológico del alantoides, el cual forma una conexión patente entre el domo vesical y el ombligo. Las anormalidades del uraco son una causa muy infrecuente de patología abdominal asociada, refiriéndose principalmente al abdomen agudo. Esta entidad es extraordinariamente inusual en la población adulta, encontrándose en el 0,063\%, y con mayor frecuencia en hombres que en mujeres. Las complicaciones asociadas van desde las infecciosas hasta las neoplásicas, y su manejo es casi siempre quirúrgico en uno o dos tiempos.

Presentamos el caso de una paciente obesa mórbida, con absceso del uraco, que causó una obstrucción intestinal a nivel de asas delgadas; resaltamos la obesidad como un factor de riesgo durante el pre, peri y el postoperatorio, y la necesidad de un abordaje multidisciplinario para un manejo exitoso.

\section{Presentación de caso}

Se trata de una paciente de sexo femenino de 59 años, con antecedente de obesidad mórbida (IMC $48 \mathrm{~kg} / \mathrm{m} 2$ ), quien consulta por cuadro clínico de ocho días, consistente en dolor abdominal en hipogastrio, tipo cólico, intensidad $6 / 10$, asociado a síntomas urinarios irritativos bajos (disuria, polaquiuria y tenesmo vesical, sin neumaturia). El uroanálisis tomado en otra institución fue sugestivo de infección, por lo que se indicó manejo antibiótico inicial con cefalexina, dado que se pensaba en una patología no complicada de foco urinario.

Al ingreso, presentó aumento de la intensidad de dolor, asociado a múltiples episodios eméticos de contenido alimentario y deposiciones diarreicas, por lo que se indicó una (TC) tomografía computarizada de abdomen con contraste, con reporte de remanente del uraco abscedado que condicionaba obstrucción intestinal, por compresión extrínseca de un asa delgada (figura 1).

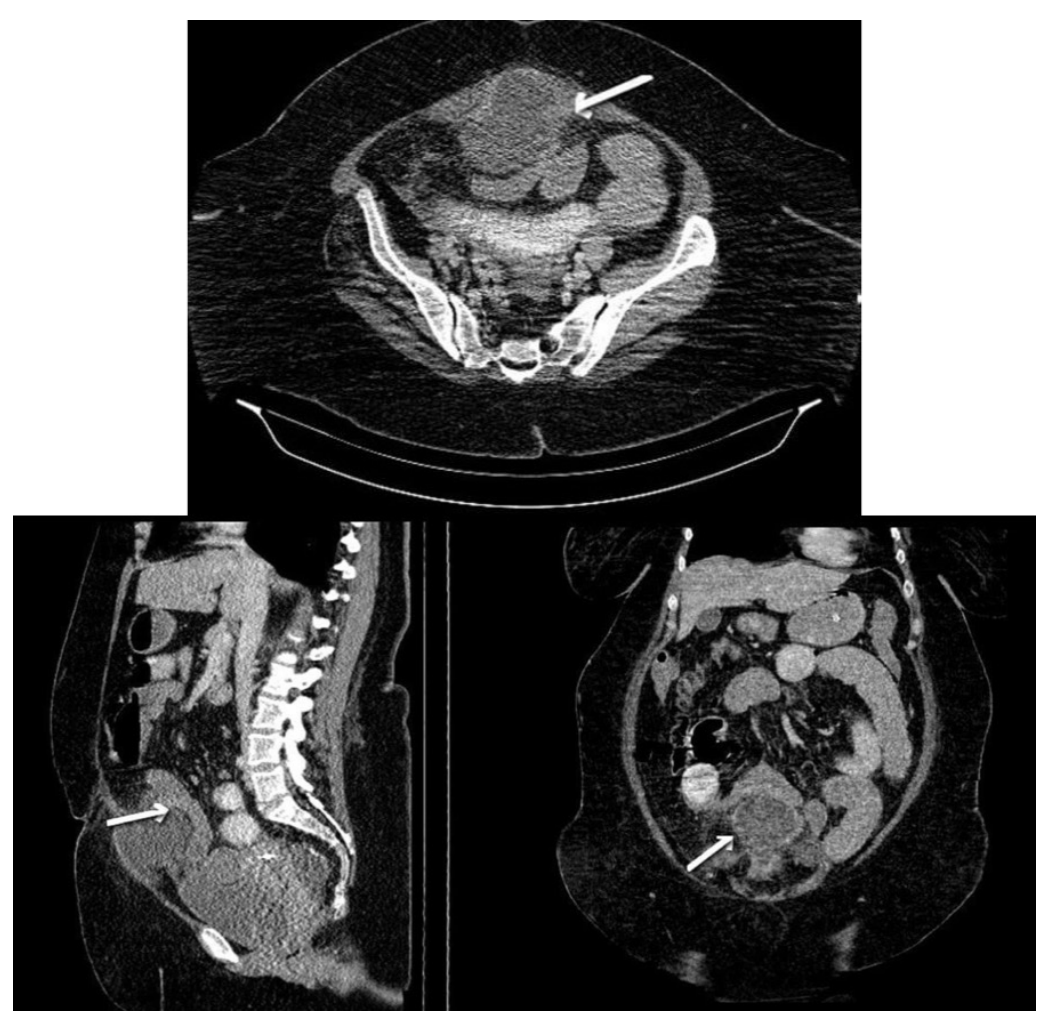

Figura 1. Tomografía computarizada de abdomen contrastada: se observa el remanente del uraco abscedado, que condiciona obstrucción intestinal por compresión extrínseca de un asa delgada. 
Se escalonó manejo antibiótico a piperacilina tazobactam y se llevó la paciente a cirugía por vía laparoscópica, ya que en las imágenes no se apreciaba distensión de asas intestinales. Se encontró una gran masa dependiente del domo vesical, ocasionando la zona de transición en el íleon terminal. Por la obesidad de la paciente no se logró una adecuada distensión abdominal, ni una visualización óptima, y se decidió conversión a laparotomía, realizando drenaje del absceso del uraco (100 ml), cistectomía parcial, resección segmentaria del íleon y anastomosis laterolateral.

Se realizó seguimiento por los servicios de cirugía general, urología y soporte nutricional, y la paciente evolucionó hacia la mejoría. El reporte del espécimen histopatológico informó un proceso inflamatorio, sin mención de malignidad. La paciente asistió a consulta ambulatoria de cirugía general y a curaciones por parte de terapia enterostomal, dado que presentó dehiscencia de la herida quirúrgica. Dos semanas después reingresó a la institución, por infección de vías urinarias complicada, asociada a bacteriemia por $E$ coli $2 B R$ e infección superficial de sitio operatorio, sin indicación de nuevo abordaje quirúrgico.

\section{Discusión}

La primera descripción de una anomalía sintomática del uraco fue realizada en 1550 por Bartholomaeus Cabrolius, quien identificó una fístula vesículo-umbilical en una paciente de 18 años con obliteración del meato urinario. Esta condición fue tratada quirúrgicamente al realizar dilatación uretral y ligadura de la protrusión umbilical. Posteriormente, desde esa fecha y hasta 1970 se reportaron 315 casos en la literatura. Sin embargo, gracias al uso rutinario de imágenes diagnósticas durante las últimas décadas, se ha encontrado un aumento en su prevalencia, y hallazgo incidental de anomalías del uraco en pacientes asintomáticos. La prevalencia de esta entidad es mayor en la población pediátrica, correspondiendo al 1,6 \% en menores de 15 años y 0,063 \% en adultos, con mayor frecuencia en hombres que en mujeres (relación 2:1), ${ }^{1}$.
El uraco es un remanente embrionario del alantoides (derivado del saco vitelino), y de la porción ventral de la cloaca (extensión del seno urogenital), el cual, en adultos, forma una conexión patente entre el domo vesical y el ombligo ${ }^{1,2}$. Entre el cuarto y quinto mes de gestación, la vejiga desciende hacia la pelvis y el lumen del alantoides se oblitera para formar el uraco, un tracto tubular fibromuscular epitelizado, el cual está localizado en el espacio de Retzius (entre la fascia transversa y el peritoneo parietal), ${ }^{1-3}$. Entre el tercer trimestre y el periodo neonatal, el uraco se oblitera, convirtiéndose así en el ligamento umbilical medial ${ }^{1,3}$. Si existe algún defecto en su involución, pueden originarse anomalías del uraco, que pueden ser congénitas o adquiridas.

Existen cuatro anomalías congénitas del uraco: uraco patente (fístula), quiste del uraco, seno umbílico-uracal y divertículo vesículo-uracal ${ }^{1-3}$. El uraco patente presenta una comunicación persistente entre la vejiga y el ombligo ${ }^{1,3-5}$. El quiste del uraco resulta de la obliteración de los extremos con un segmento focal remanente, usualmente localizado en el tercio inferior del uraco, sin conexión directa con la vejiga ni el ombligo ${ }^{1,3}$. El seno del uraco es una estructura quística que se conecta con el ombligo, y el divertículo vesículo-uracal, que presenta una apertura patente hacia la vejiga ${ }^{3,6}$. Los remanentes del uraco en lactantes menores de seis meses suelen resolver de forma espontánea, sin necesidad de intervención quirúrgica ${ }^{3}$.

Las anomalías adquiridas del uraco corresponden a las complicaciones infecciosas y al desarrollo de malignidad ${ }^{1,3}$. Cualquiera de las anomalías congénitas puede sobre infectarse por vía hematógena, linfática o vesical (estasis urinaria crónica). Los microorganismos más comunes encontrados en los cultivos son S. aureus, E. coli, Enterococcus, Citrobacter, Klebsiella y Proteus. Si el compromiso infeccioso es severo, este puede progresar a abscesos, los cuales tienen potencial de producir peritonitis y sepsis, en caso de ruptura intraperitoneal ${ }^{1}$.

Dentro de las neoplasias malignas del uraco, la más común es el adenocarcinoma mucinoso del uraco (42\%), seguido del adenocarcinoma no mucinoso (30\%), el carcinoma de células de 
transición (10 \%) y el carcinoma de células escamosas. Se han reportado casos de carcinoma del uraco metastásico ${ }^{5}$. La transformación maligna es poco frecuente, representando menos del $1 \%$ de las neoplasias de la vejiga ${ }^{1}$,y ocurre en adultos mayores entre la quinta y la séptima década de la vida ${ }^{5,7,8}$.

Dada la localización anatómica del uraco, el cuadro clínico de estas patologías suele ser inespecífico o silente ${ }^{6}$. No obstante, las manifestaciones clásicas de la infección del uraco son el dolor abdominal (periumbilical o generalizado), el eritema periumbilical, masa palpable, la excreción umbilical de orina (característico de uraco patente), los síntomas irritativos urinarios, los episodios de retención urinaria y fiebre ${ }^{1,3,6}$. La hematuria ha sido reportada en pacientes con neoplasias malignas del uraco ${ }^{5}$.

A menos que exista drenaje de orina a través del ombligo, la poca especificidad del cuadro clínico, obliga a descartar otras entidades abdominales más frecuentes, como apendicitis aguda, infección urinaria recurrente, prostatitis aguda, hernia umbilical estrangulada, enfermedad inflamatoria intestinal, enfermedad pélvica inflamatoria, tumores de la pared abdominal y divertículo de Meckel ${ }^{3-9}$. A su vez, las anomalías del uraco pueden dar lugar a adherencias, inflamación y obstrucción intestinal mecánica ${ }^{8}$.

Es necesario el uso de ayudas diagnósticas para identificar y caracterizar las anomalías del uraco. La evaluación diagnóstica con imágenes suele limitarse al ultrasonido y la tomografía computarizada ${ }^{6-9,10-12}$. El ultrasonido abdominal es la modalidad de primera elección y dependiendo del tipo de anomalía, logra el diagnóstico en el $90 \%$ de los casos, ya que el uraco se localiza en la pared abdominal anterior y no existe interferencia de estructuras intestinales ${ }^{3,9}$. Se identifica como una masa inflamatoria, hipoecoica y extraperitoneal, fija, localizada en la línea media, en la superficie anterosuperior de la vejiga ${ }^{2,10}$. Adicionalmente, se puede evidenciar un absceso dentro del tejido celular subcutáneo de la pared abdominal (localizado en el espacio extraperitoneal) con extensión a la región umbilical ${ }^{9}$.
En caso de que los hallazgos identificados en ultrasonido no sean concluyentes o se tenga sospecha de malignidad, se recomienda la TC como imagen de segunda opción ${ }^{7,11}$. La tomografía logra identificar con mayor facilidad el tipo de anomalía, en caso de un absceso, se puede visualizar una estructura de forma cónica, localizada posterior al músculo recto abdominal, con cambios inflamatorios de la grasa mesentérica ${ }^{2,10}$. Además, permite evaluar las estructuras y órganos adyacentes con el fin de descartar otras patologías abdomino-pélvicas ${ }^{12}$.

No obstante, resulta complicado diferenciar entre una lesión benigna y cáncer del uraco ${ }^{8}$, los abscesos del uraco pueden confundirse con carcinomas, ya que se presentan como una masa sólida de bordes gruesos e irregulares ${ }^{5}$. Existen algunas características imagenológicas sugestivas de malignidad $^{5,10}$, como las calcificaciones (50-70 \%) y la invasión a la pared vesical (92\%), ${ }^{5}$. Si existe sospecha de malignidad, se debe realizar cistoscopia con biopsia y citología urinaria ${ }^{9,12}$.

La resonancia magnética nuclear (RMN) permite tener una mejor visualización de los tejidos blandos y evaluar la extensión local, el absceso del uraco se identifica como una masa hipointensa en T1 y captación heterogénea tras la administración de gadolinio ${ }^{5}$. Los pacientes con obesidad representan un mayor reto para la identificación de remanentes del uraco en estudios imagenológicos. La laparoscopia diagnóstica tiene gran utilidad si las imágenes diagnósticas son inconclusas, permitiendo, además la resección quirúrgica del mismo ${ }^{13,14}$.

A pesar de que la historia natural de estas anomalías es desconocida y poco estudiada, se ha identificado el riesgo de transformación maligna durante la edad adulta ${ }^{15}$. En los niños esta transformación es muy rara, y por el contrario, varios reportes han identificado la regresión espontánea de las anomalías del uraco ${ }^{1,4}$.

El manejo de patologías del uraco en pacientes sintomáticos depende de la edad al momento del diagnóstico ${ }^{1,7}$. Actualmente los lactantes menores de 1 año reciben manejo médico y posterior observación con ecografías seriadas para determinar el 
cierre definitivo del defecto, en caso de presentar síntomas recurrentes o fallo terapéutico, se debe considerar la resección quirúrgica ${ }^{1}$. El manejo definitivo en pacientes sintomáticos es quirúrgico, lo cual disminuye la posibilidad de recurrencia o de transformación maligna ${ }^{16}$.

En cuanto a las anomalías congénitas no complicadas, se recomienda la resección completa del remanente, mediante un abordaje quirúrgico primario tradicional, que consiste en una incisión infraumbilical transversa, o mediante laparoscopia, con posterior resección del tracto desde la base del ombligo hasta el domo de la vejiga ${ }^{1,3,13,14}$. El abordaje abierto se ha asociado con aumento de la morbilidad, mayores complicaciones, mayor duración del acto quirúrgico y mayor estancia hospitalaria, por lo que un abordaje mínimamente invasivo en el manejo de patologías del uraco se ha convertido en una opción adecuada, especialmente en pacientes con obesidad mórbida ${ }^{13}$.

La primera escisión de un remanente del uraco por vía laparoscópica fue realizada por Trondsen et al., en 1993, desde entonces, se han realizado varios reportes y series de caso de diferentes tipos de remanentes, los cuales han tenido resultados favorables similares, demostrando la efectividad de la resección completa del uraco, asociado a disminución de la morbilidad y las complicaciones, tales como la recurrencia y la dificultad para la micción ${ }^{13,17}$. A pesar de los beneficios de la vía laparoscópica en comparación con el abordaje abierto, se ha documentado un leve riesgo de lesión vesical, especialmente en el contexto de inflamación severa ${ }^{18}$.

Las anomalías congénitas que se extienden a la vejiga (uraco patente y divertículo vesículo-uracal), requieren un abordaje más agresivo, con resección de la cúpula vesical, para prevenir complicaciones, entre ellas la transformación maligna ${ }^{11,16,19}$.

En pacientes con complicaciones infecciosas, se recomienda realizar un abordaje en dos etapas, el manejo inicial incluye cubrimiento antibiótico de amplio espectro de manera temprana y drenaje de la colección purulenta ${ }^{1,3}$. Posterior a la resolución del cuadro inflamatorio, se realiza la resección completa del remanente ${ }^{7}$. En caso de un absceso del uraco, se prefiere la resección quirúrgica de la masa y cistectomía parcial ${ }^{4}$. En el caso presentado, se tomó la decisión de realizar un abordaje agresivo con cistectomía parcial debido al importante compromiso vesical causado por el proceso infeccioso del remanente del uraco. La realización de la onfalectomía es debatida y depende del compromiso de los tejidos adyacentes o de la presencia de malignidad ${ }^{13}$.

En caso de cáncer del uraco, se debe considerar la cistectomía radical o parcial, asociada a la resección en bloque de los tejidos del uraco, la fascia posterior de la vaina de los rectos y linfadenectomía pélvica bilateral ${ }^{3,12,20}$. El pronóstico es poco favorable y la utilidad de radio o quimioterapia no es clara, debido a que estos tumores son considerados resistentes ${ }^{5,7}$.

En pacientes asintomáticos, existe controversia en cuanto al abordaje quirúrgico de los remanentes del uraco ${ }^{4,7}$. Naiditch et. al., condujeron un estudio retrospectivo en población pediátrica, encontrando que los remanentes del uraco diagnosticados incidentalmente pueden ser manejados médicamente sin desarrollo de complicaciones a corto plazo, con una alta probabilidad de autorresolución, ocho de 18 pacientes asintomáticos fueron observados durante un intervalo de tiempo de 9,6 meses desde el diagnóstico inicial, con resolución espontánea en siete de ellos ${ }^{4}$. En cuanto al seguimiento, faltan estudios para determinar el abordaje clínico más adecuado, con el fin de prevenir la recurrencia y detectar complicaciones posoperatorias de manera precoz.

\section{Conclusión}

Las anormalidades y la presentación clínica de este caso, ofrecen múltiples aproximaciones al manejo ideal. Las vías de acceso y el drenaje de la colección fueron idóneos para lograr la resolución de la patología presentada. Es importante resaltar el abordaje individualizado de cada paciente, teniendo en cuenta sus comorbilidades, los hallazgos imagenológicos e intraoperatorios y las complicaciones derivadas de esta patología. Los pacientes deben recibir un manejo integral, para lograr la resolución de los síntomas, y prevenir su recurrencia. 


\section{Cumplimiento de normas éticas}

Consentimiento informado: Se obtuvo el consentimiento informado por parte del paciente.

Conflicto de interés: Ninguno declarado por los autores.

Financiación: este trabajo fue autofinanciado.

\section{Contribución de los autores:}

Concepción y diseño del estudio: Paulo Cabrera, Lina Sanabria.

Adquisición de datos: Carlos Pérez, Sara Sofía Avendaño, Paula Sanabria.

Análisis e interpretación de datos: Paulo Cabrera, Lina Sanabria, Carlos Pérez, Sara Sofía Avendaño, Paula Sanabria.

Redacción del manuscrito: Paulo Cabrera, Lina Sanabria, Carlos Pérez, Sara Sofía Avendaño, Paula Sanabria.

Revisión crítica: Paulo Cabrera, Lina Sanabria, Carlos Pérez, Sara Sofía Avendaño, Paula Sanabria.

\section{Referencias}

1. Parada C, Adam SZ, Nikolaidis P, Yaghmai V, Miller FH. Imaging of the urachus: anomalies, complications, and mimics. RadioGraphics. 2016;36:2049-2063. https://doi.org/10.1148/rg.2016160062

2. Aboitiz CM. A propósito del absceso del uraco. Presentación de un caso y revisión de la literatura. Rev Mex Pediatr. 1999;66:254-6.

3. Garcia M de Q Barral CM. Patent urachus presenting as acute abdomen. J Med Ultrason. 2015;23:189-92. https://doi.org/10.1016/j.jmu.2015.08.003

4. Naiditch JA, Radhakrishnan J, Chin AC. Current diagnosis and management of urachal remnants. J Pediatr Surg. 2013;48:2148-52. https://doi.org/10.1016/j.jpedsurg.2013.02.069

5. Lam SW, Linsen PV, Elgersma OE. Infection of previously closed urachus mimicking malignancy: a case report and literature review of radiological findings to the diagnosis. Clin Med Insights Case Reports. 2019;12:1179547619843836. https://doi.org/10.1177/1179547619843836

6. Nimonrat A, Na-ChiangMai W, Mutarak M. Urachal abnormalities: clinical and imaging features. Singapore Med J. 2008;49:930-5.

7. Tazi F, Ahsaini M, Khalouk A, Mellas S, Stuurman-Wieringa RE, Elfassi MJ, et al. Abscess of urachal remnants presenting with acute abdomen: a case series. J Med Case Rep. 2012;6:226.

https://doi.org/10.1186/1752-1947-6-226

8. O'Leary MP, Ashman ZW, Plurad DS, Kim DY. Urachal cyst causing small bowel obstruction in an adult with a virgin abdomen. Case Reports in Surgery, 2016;7:1-4. https://doi.org/10.1155/2016/3247087

9. Muśko N, Dobruch J, Piotrowicz S, Szostek P, Borówka A. Infected urachal cyst in a young adult. Cent European J Urol. 2014;67:199-201. https://doi.org/10.5173/ceju.2014.02.art19

10. Ramos-Pacheco VH, Saldaña-Dominguez Y, Cervantes-Sánchez AC. Infected urachal remnants: an unusual presentation. BJR Case Rep. 2016;2:20150226. https://doi.org/10.1259/bjrcr.20150226

11. Ponce-Pérez L, Cárdenas-Lailson L, Domínguez-Muñoz G, López-Díaz Y, Vera-Rodríguez F. Quiste de uraco gigante abscedado en adulto. Reporte de un caso. Cir Cir. 2013;81:348-52.

12. Donate-Moreno MJ, Giménez-Bachs JM, Salinas-Sánchez AS, Lorenzo-Romero JG, Segura-Martín M, Hernández-Millán I, et al. Patología del uraco: revisión de conjunto y presentación de tres casos. Actas Urol Esp. 2005;29:332-6. https://doi.org/10.1016/S0210-4806(05)73249-6

13. Sánchez-Ismayel A, Cruz-González G, Sánchez R, Sánchez-Salas R, Rodríguez O, Sanabria E, et al. Manejo laparoscópico de las anomalías sintomáticas del uraco. Actas Urol Esp. 2009;33:284-9. https://doi.org/10.1016/S0210-4806(09)74142-7

14. Reznichenko A. Minimally invasive approach to urachal remnant in obese patient. J Curr Surg. 2014;4:117-20. https://doi.org/10.14740/jcs245w

15. Ashley R, Inman B, Routh J, Rohlinger A, Husmann D, Kramer S. Urachal anomalies: a longitudinal study of urachal remnants in children and adults. J Urol. 2007;178(4S):1615-8. https://doi.org/10.1016/j.juro.2007.03.194

16. Martín-Hernández D, Prieto-Matos P, Díez-Hernández JC, Liras-Muñoz J, Celis-Villasana L de. Persistencia de uraco infectado como causa de abdomen agudo: A propósito de un caso. Arch Esp Urol. 2009;62:589-92. https://doi.org/10.4321/S0004-06142009000700010

17. Jeong H, Han D, Kwon W. Laparoscopic management of complicated urachal remnants. Chonnam Med J. 2013;49:43. https://doi.org/10.4068/cmj.2013.49.1.43

18. Sukhotnik I, Aranovich I, Mansur B, Matter I, Kandelis Y, Halachmi S. Laparoscopic surgery of urachal anomalies: a single-center experience. Isr Med Assoc J. 2016;18:673-6.

19. Yiee J, Garcia N, Baker L, Barber R, Snodgrass W, Wilcox D. A diagnostic algorithm for urachal anomalies. J Pediatr Urol. 2007;3:500-4. https://doi.org/10.1016/j.jpurol.2007.07.010

20. Moreno-Bencardino, C, Fernández-Bonilla, N, Peñaloza, I, Meek, E, Torres-Castellanos, L. Gran adenocarcinoma de uraco. Reporte de un caso y revisión de la literatura. Urol Colomb. 2017;26: 239-45. http://dx.doi.org/10.1016/j.uroco.2017.03.001 